\title{
Enhanced recovery after surgery nursing program, a protective factor for stoma-related complications in patients with low rectal cancer
}

\author{
Weiling Shao ${ }^{1}$, Honggang Wang ${ }^{1}$, Qun Chen ${ }^{1}$, Wen Zhao ${ }^{1}$, Yulian Gu ${ }^{1}$ and Guoqin Feng ${ }^{2 *}$
}

\begin{abstract}
Background: This study aimed to investigate the association between enhanced recovery after surgery (ERAS) nursing program and stoma-related complications (SRCs) and prognosis in patients with low rectal cancer (LRC) undergoing abdominoperineal resection with sigmoidostomy.

Methods: LRC patients who underwent elective abdominoperineal resection with sigmoidostomy between May 2016 and May 2019 were retrospectively enrolled. The occurrence of early major or minor SRCs (within postoperative 30 days) was set as the primary end-point. Clinicopathological variables and laboratory tests were compared between patients with or without SRCs. The univariate and multivariate logistic regression analyses were performed to investigate risk factors for SRCs. Hospitalization satisfaction-related and prognosis-related variables were compared between LRC patients with or without ERAS nursing program.

Results: A total of 288 patients were enrolled and the incidence of SRCs was $26.7 \%(77 / 288)$. ERAS nursing program was the only independent risk factor for SRCs in LRC patients (OR 2.04,95\% Cl 1.31-3.12, P=0.016) by the multivariate logistic regression analysis. Moreover, ERAS nursing program was associated with higher hospitalization satisfaction rate, faster bowel function recovery, better psychological status, and higher quality of life.
\end{abstract}

Conclusions: ERAS nursing program was a protective factor for SRCs and associated with improved prognosis in LRC patients undergoing elective abdominoperineal resection with sigmoidostomy.

Keywords: Low rectal cancer, Stoma-related complications, Enhanced recovery after surgery nursing program, Prognosis, Life of quality

\section{Background}

Colorectal cancer (CRC) is the third most common cancer and the second leading cause of cancer-related death worldwide with over 1.8 million new cases and 881,000 cancer-related death in 2018 [1]. The incidence of low rectal cancer (LRC) among Chinese is reported to be 60-75\% higher than Western populations [2, 3]. As for those LRC patients, abdominoperineal resection with

\footnotetext{
*Correspondence: fengguoqin.tz@outlook.com

2 Department of Nursing, Taizhou People's Hospital, No. 366 Taihu Road,

Taizhou 225300, Jiangsu Province, China

Full list of author information is available at the end of the article
}

sigmoidostomy is widely accepted as an effective and safe surgical strategy and considered as the standard treatment to decrease the risk of positive distal edge [4]. A stoma is a physical and psychological burden for the patients to accept this anatomical change. The reported rates of stoma-related complications (SRCs) are quite high, ranging from 21 to $70 \%$ [5, 6]. Furthermore, its real frequency is probably underestimated due to the autonomous management by stoma therapist without records. SRCs may have a great impact on the postoperative recovery, morbidity, mortality, and quality of life (QOL) [7-9]. Therefore, to investigate potential predictors and 
effective preventive measures for SRCs is of great clinical importance for prognosis improvement.

Enhanced recovery after surgery (ERAS), which was first put forward by Kehlet in the mid-1990s [10], has shown notably promising results in reducing the length of stay (LOS) and morbidity after colorectal surgery $[11,12]$. A recent meta-analysis by Lau et al. has demonstrated that ERAS is associated with decreased LOS, postoperative complications, economic cost, and earlier gastrointestinal function recovery in colorectal surgery [13]. Nursing is a critically important part of ERAS and it is becoming more popular in clinical application. However, the predictive role of ERAS nursing program for SRCs remains unclear, which was the main goal of this present study.

\section{Methods}

\section{Patients}

This was a single-center retrospective study and it was approved by the Medical Institutional Ethics Committee of our hospital. We retrospectively recruited LRC patients who underwent elective abdominoperineal resection with sigmoidostomy at the Department of General Surgery, Taizhou Peoples' Hospital between May 2016 and May 2019. Each patient was required to offer the signed informed consent. Inclusion criteria: (a) aged between 18 and 75 years; (b) with a histological diagnosis with LRC (within $8 \mathrm{~cm}$ from the anal verge); (c) abdominoperineal resection with sigmoidostomy. Exclusion criteria: (a) with surgical contraindication, e.g. distant metastasis, and severe obstruction; (b) undergoing emergency operations; (c) with malignancies in other systems; (d) with severe hematological, hepatic, kidney disorder, or autoimmune diseases; (e) with preoperative chemotherapy, radiotherapy, or targeted therapy for cancers; (f) with incomplete data. All the patients were under the perioperative management of the same surgical, anesthesia, and nursing team. Protocols of ERAS nursing program were according to the latest Chinese guidelines of ERAS (version 2016 and 2018). In brief, preoperative ERAS program education, personalized nutritional evaluation and support (based on NRS 2002 score), psychological counseling, psychological status evaluation, and improvement (reduce anxiety), no overnight fasting (clear fluids up to $2 \mathrm{~h}$ and solid food up to $6 \mathrm{~h}$ before the induction of anaesthesia) and carbohydrate loading drinks before the surgery $(10 \%$ glucose solution oral administered $1000 \mathrm{ml} 10 \mathrm{~h}$ and $400 \mathrm{ml} 2 \mathrm{~h}$ before the induction of anaesthesia), preoperative bowel preparation (polyethylene glycol electrolyte powder the night before the surgery), venous thromboembolism prevention (based on Caprini score), intraoperative warming (maintain core body temperature $\geq 36{ }^{\circ} \mathrm{C}$ ), avoidance of routine abdominal drains and nasogastric tubes, encouraged early postoperative mobilization (postoperative day 1 ), early catheter remove (postoperative day 1), dietary (fluids and enteral nutrition from postoperative day 1), pain (multimodal analgesis, combined with NSAIDs, reduce the use of opioid analgesics), sleep, and psychology management were recommended. Enrolled patients were followed up for at least three months from the surgical day.

The following data were extracted from our database and recorded: (1) demographic variables including age, gender, body mass index (BMI), smoking and drinking habits; (2) clinical baseline variables including serum carcinoembryonic antigen (CEA), American Society of Anesthesiologists (ASA) grade, Charlson Comorbidity Index [14], nutritional risk score (NRS)-2002 [15]; (3) pathological data including $\mathrm{T}$ stage, $\mathrm{N}$ stage, TNM stage, and pathologic differentiation; (4) treatment-related variables including surgical approach, ERAS nursing program, preoperative stoma localization, operation time, estimated blood loss, height of stoma, and base area of stoma; (5) laboratory tests including hemoglobin $(\mathrm{Hb})$, albumin (Alb), white blood cell (WBC), hematocrit (Hct), C-reactive protein (CRP), creatinine, and urea; (6) Chinese hospitalization satisfaction-related variables (11 items) including safety, environment, accessibility, respect, nursing technique, comfortableness, health education, communication, emotion support, participation in nursing, and discharge and referral; (7) prognosis-related variables including time to first exhaust and defecation, LOS, self-rating anxiety scale (SAS) scores, self-rating depression scale (SDS) scores [16], Gastrointestinal Quality of Life Index (GIQLI) [17] including physiological function, mental health, social function, and subjective symptoms at 3 months after the surgery.

As described by previous reports, the primary observational end-point was the occurrence of early major or minor SRCs (within postoperative 30 days). In brief, major SRCs included stoma prolapse, parastomal hernia, stricture, fistula, retraction, ischemia, and bleeding. Minor SRCs included skin alterations according to the classification by SACS ${ }^{\mathrm{TM}}$ instrument (ConvaTec, Reading, Berkshire, UK) for assessing peristomal skin lesion [18].

The base area of stoma was measured postoperatively by nurses calculating from the horizontal and vertical size of the stoma base. The pathological TNM stage was identified according to the criteria by the American Joint Committee on Cancer/International Union Against Cancer (7th edition).

\section{Statistical analysis}

GraphPad Prism 8.0 (GraphPad Inc., CA, USA) and SPSS 19.0 (SPSS Inc., Chicago, IL, USA) were used for 
data analysis. Data are expressed as number (n) with percentage (\%) or mean with standard deviation (SD) as appropriate. Data are analyzed using the Chi-square test, Student's $t$-test, and Mann-Whitney U-test as appropriate. The univariate and multivariate logistic regression analyses were performed to investigate risk factors for SRCs. A two-sided P value $<0.05$ was considered statistically different.

\section{Results}

\section{Patient characteristics}

Three hundred and thirty-three LRC patients who underwent elective abdominoperineal resection with sigmoidostomy were initially enrolled. Forty-five were excluded due to the following reasons (10 with surgical contraindication, 6 undergoing emergency operations, 5 with malignancies in other systems, 9 with severe hematological, hepatic, or kidney disorder, and 15 with incomplete data) and two hundred and eighty-eight subjects were included in the analysis. The mean age of the cohort was 66.2 years and the majority $(69.4 \%, 200 / 288)$ were male patients. The total incidence of SRCs was $26.7 \%(77 / 288)$. The clinicopathological characteristics associated with SRCs are shown in Table 1. There were no significant differences in gender, ASA grade, smoking and drinking habits, serum CEA, T stage, N stage, TNM stage, pathologic differentiation, surgical approach, estimated blood loss, and height of stoma between patients with and without SRCs $(P>0.05)$. Patients in the SRCs group seemed to have a higher percentage of elderly ( $\geq 65$ years) $(\mathrm{P}=0.048)$ and overweight $(\mathrm{BMI}>24.5) \quad(\mathrm{P}=0.031)$. Patients with a higher NRS 2002 score $(\geq 3)(P=0.015)$, the base area of stoma $(\mathrm{P}=0.019)$, and longer operation time $(\mathrm{P}=0.028)$ were associated with SRCs occurrence. Moreover, significant differences were noted for ERAS nursing program $(\mathrm{P}=0.010)$ and preoperative stoma localization $(\mathrm{P}=0.040)$ between patients who developed SRCs or not.

\section{Laboratory variables associated with SRCs}

Table 2 presents the preoperative levels of laboratory variables in patients with or without SRCs. The results indicated that patients with SRCs had higher rates of abnormal Alb $(<35.0 \mathrm{~g} / \mathrm{L})$ and CRP $(>0.8 \mathrm{mg} / \mathrm{L})$ expressions $(\mathrm{P}=0.025$ and 0.038 , respectively). No statistically significant differences were observed concerning $\mathrm{Hb}$, WBC, creatinine, and urea between these two groups $(\mathrm{P}>0.05)$.

\section{Risk factors for SRCs}

To identify potential risk factors for SRCs, the univariate and multivariate logistic regression analyses were performed. As illustrated in Table 3, five variables
Table 1 Clinicopathological variables associated with SRCs in LRC patients

\begin{tabular}{|c|c|c|c|}
\hline \multirow[t]{2}{*}{ Variables } & \multicolumn{2}{|l|}{ SRCs } & \multirow[t]{2}{*}{$P$-value } \\
\hline & Yes & No & \\
\hline Number, n (\%) & $77(26.7)$ & $211(73.3)$ & - \\
\hline Age (year), n (\%) & - & - & $0.048^{*}$ \\
\hline$\geq 65$ & $47(61.0)$ & $101(47.9)$ & - \\
\hline$<65$ & $30(39.0)$ & $110(52.1)$ & - \\
\hline Gender, n (\%) & - & - & 0.114 \\
\hline Male & $48(62.3)$ & $152(72.0)$ & - \\
\hline Female & $29(37.7)$ & $59(28.0)$ & - \\
\hline BMI $\left(\mathrm{kg} / \mathrm{m}^{2}\right), \mathrm{n}(\%)$ & - & - & $0.031^{*}$ \\
\hline$\geq 24.5$ & $31(40.3)$ & $57(27.0)$ & - \\
\hline$<24.5$ & $46(59.7)$ & $154(73.0)$ & - \\
\hline ASA grade, n (\%) & - & - & 0.668 \\
\hline$|/| \mid$ & $49(63.6)$ & $140(66.4)$ & - \\
\hline III/IV & $28(36.4)$ & 71 (33.6) & - \\
\hline Charlson Comorbidity Index & $3.2 \pm 0.8$ & $2.5 \pm 0.6$ & $<0.001$ \\
\hline NRS 2002 score, n (\%) & - & - & $0.015^{*}$ \\
\hline$\geq 3$ & $17(22.1)$ & $23(10.9)$ & \\
\hline$<3$ & $60(77.9)$ & $188(89.1)$ & \\
\hline Active smoker, n (\%) & $10(13.0)$ & $26(12.3)$ & 0.880 \\
\hline Heavy drinker, n (\%) & $8(10.4)$ & $22(10.4)$ & 0.993 \\
\hline Serum CEA (ng/mL), n (\%) & - & - & 0.686 \\
\hline$\geq 5$ & $36(46.8)$ & $93(44.1)$ & - \\
\hline$<5$ & $41(53.2)$ & $118(55.9)$ & - \\
\hline T stage, n (\%) & - & - & 0.409 \\
\hline $\mathrm{T} 1 / 2$ & $23(29.9)$ & $74(35.1)$ & - \\
\hline $\mathrm{T} 3 / 4$ & $54(70.1)$ & $137(64.9)$ & - \\
\hline N stage, n (\%) & - & - & 0.163 \\
\hline Negative & $45(58.4)$ & $142(67.3)$ & - \\
\hline Positive & 32 (41.6) & 69 (32.7) & - \\
\hline TNM stage, n (\%) & - & - & 0.663 \\
\hline$|/| \mid$ & $46(59.7)$ & $120(56.9)$ & - \\
\hline III & $31(40.3)$ & $91(43.1)$ & - \\
\hline Pathologic differentiation, n (\%) & - & - & 0.936 \\
\hline Well/moderate & $62(80.5)$ & $169(80.1)$ & - \\
\hline Poor/mucinous & $15(19.5)$ & $42(19.9)$ & - \\
\hline Surgical approach, n (\%) & - & - & 0.371 \\
\hline Laparoscopic & $54(70.1)$ & $159(75.4)$ & - \\
\hline Laparotomy & $23(29.9)$ & $52(24.6)$ & - \\
\hline ERAS nursing program, n (\%) & - & - & $0.010^{*}$ \\
\hline Yes & $41(53.2)$ & $147(69.7)$ & - \\
\hline No & $36(46.8)$ & 64 (30.3) & - \\
\hline $\begin{array}{l}\text { Preoperative stoma localization, } \\
\text { n (\%) }\end{array}$ & $10(13.0)$ & $51(24.2)$ & $0.040^{*}$ \\
\hline Operation time (min) & $167.3 \pm 37.4$ & $155.9 \pm 39.3$ & $0.028^{*}$ \\
\hline Estimated blood loss (ml) & $138.1 \pm 50.5$ & $146.5 \pm 55.7$ & 0.247 \\
\hline Height of stoma (mm) & $10.7 \pm 1.3$ & $11.0 \pm 1.2$ & 0.067 \\
\hline Base area of stoma $\left(\mathrm{cm}^{2}\right)$ & $9.0 \pm 1.1$ & $8.7 \pm 0.9$ & $0.019^{*}$ \\
\hline
\end{tabular}

ERAS enhanced recovery after surgery, $L R C$ low rectal cancer, $S R C S$ stoma-related complications, $B M I$ body mass index, ASA American Society of Anesthesiologists, $N R S$ nutritional risk score, $C E A$ carcinoembryonic antigen

*P value $<0.05$ 
Table 2 Laboratory tests associated with SRCs in LRC patients

\begin{tabular}{lllc}
\hline Variables & \multicolumn{2}{l}{ SRCs } & P-value \\
\cline { 2 - 3 } & Yes & No & \\
\hline Number, $\mathrm{n}(\%)$ & $77(26.7)$ & $211(73.3)$ & - \\
$\mathrm{Hb}(\mathrm{g} / \mathrm{L})$ & $109.7 \pm 7.7$ & $110.3 \pm 8.1$ & 0.722 \\
$\mathrm{WBC}\left(\times 10^{9} / \mathrm{L}\right)$ & $7.5 \pm 2.0$ & $7.1 \pm 1.8$ & 0.107 \\
Albumin $(\mathrm{g} / \mathrm{L}), \mathrm{n}(\%)$ & - & - & $0.025^{*}$ \\
$<35.0$ & $21(27.3)$ & $33(15.6)$ & \\
$\geq 35.0$ & $56(72.7)$ & $178(84.4)$ & \\
CRP $(\mathrm{mg} / \mathrm{L}), \mathrm{n}(\%)$ & - & - & $0.038^{*}$ \\
$>0.8$ & $33(42.9)$ & $63(29.9)$ & - \\
$\leq 0.8$ & $44(57.1)$ & $148(70.1)$ & - \\
Creatinine(umol/L) & $71.8 \pm 9.8$ & $72.5 \pm 10.1$ & 0.600 \\
Urea(mmol/L) & $6.0 \pm 1.3$ & $5.9 \pm 1.2$ & 0.541 \\
\hline
\end{tabular}

$L R C$ low rectal cancer, SRCs stoma-related complications, $\mathrm{Hb}$ hemoglobin, Alb albumin, WBC white blood cell, Hct hematocrit, CRP C-reactive protein

${ }^{*} P$ value $<0.05$

(age, NRS 2002 score, ERAS nursing program, Alb, and CRP) were risk factors associated with SRCs $(\mathrm{P}<0.05)$. Our results from the multivariate logistic regression analysis indicated ERAS nursing program as the only independent risk factor for SRCs in LRC patients (OR $2.04,95 \%$ CI $1.31-3.12, \mathrm{P}=0.016)$.

\section{Hospitalization satisfaction-related variables and ERAS nursing program}

Based on the presence of ERAS nursing program, enrolled patients were categorized into two groups (188 with ERAS nursing program and 100 without). The eleven hospitalization satisfaction-related variables were compared between patients with or without ERAS nursing program. As shown in Table 4, patients who underwent ERAS nursing program had higher scores of 4 items (comfortableness, communication, emotional support, and participation in nursing) than those without ERAS nursing program $(\mathrm{P}<0.05)$.

\section{Prognosis-related variables and ERAS nursing program}

The prognosis-related variables between ERAS nursing and non-ERAS nursing program groups are listed in Table 5. The time to first exhaust $(P=0.036)$ and defecation $(\mathrm{P}=0.002)$, and $\operatorname{LOS}(\mathrm{P}=0.007)$ of patients in the ERAS nursing program group were all significantly lower than those in the non-ERAS nursing program group. Moreover, patients who underwent ERAS nursing program seemed to have better psychological statuses (higher SAS and SDS scores) and higher quality of life (higher GIQLI scores) than those who did not undergo ERAS nursing program $(\mathrm{P}<0.05)$.

\section{Discussion}

To our knowledge, this was the first study to indicate ERAS nursing program as an independent risk factor for SRCs. The operation of stoma formation is commonly performed for patients with malignancy and inflammatory bowel disease (IBD) [19]. Stoma formation is usually performed after a long and complex surgical operation. This procedure is simply undertaken, but it is associated with significant morbidity, complex and life-threatening consequences [20]. The incidence of SRCs in LRC patients after abdominoperineal resection with sigmoidostomy was demonstrated to be $26.7 \%$ in our cohort. A previous single-center prospective study by Pearson et al. have reported an overall SRCs rate of $23.5 \%$ among 408

Table 3 Risk factors associated with SRCs in LRC patients by univariate and multivariate logistic regression analyses

\begin{tabular}{|c|c|c|c|c|}
\hline \multirow[t]{2}{*}{ Variables } & \multicolumn{4}{|c|}{ Univariate multivariate } \\
\hline & OR $(95 \% \mathrm{Cl})$ & $P$ value & OR $(95 \% \mathrm{Cl})$ & $P$ value \\
\hline Age $(\geq 65$ vs <65) & $1.75(1.09-2.84)$ & $0.014^{*}$ & $1.27(0.82-1.98)$ & 0.241 \\
\hline $\mathrm{BMI}(\geq 24.5$ vs $<24.5)$ & $2.31(0.72-7.23)$ & 0.169 & & \\
\hline Charlson Comorbidity Index (III vs II) & $2.10(0.75-5.51)$ & 0.121 & & \\
\hline NRS 2002 score $(\geq 3$ vs $<3)$ & $1.46(1.02-2.05)$ & $0.028^{*}$ & $1.03(0.95-1.12)$ & 0.304 \\
\hline ERAS nursing program (no vs yes) & $2.47(1.42-4.01)$ & $0.009^{*}$ & $2.04(1.31-3.12)$ & $0.016^{*}$ \\
\hline Preoperative stoma localization (no vs yes) & $1.11(0.81-1.57)$ & 0.489 & & \\
\hline Operation time $(\geq 162$ vs < 162) & $1.50(0.33-6.41)$ & 0.573 & & \\
\hline Base area of stoma ( $\geq 8.8$ vs $<8.8$ ) & $1.68(0.37-7.71)$ & 0.467 & & \\
\hline Albumin $(<35.0$ vs $\geq 35.0)$ & $1.78(1.04-2.89)$ & $0.012^{*}$ & $2.05(0.65-6.49)$ & 0.221 \\
\hline $\mathrm{CRP}(>0.8 \mathrm{vs} \leq 0.8)$ & $1.54(1.01-2.35)$ & $0.037^{*}$ & $1.26(0.93-1.71)$ & 0.122 \\
\hline
\end{tabular}

ERAS enhanced recovery after surgery, $L R C$ low rectal cancer, $S R C S$ stoma-related complications, $B M I$ body mass index, NRS nutritional risk score, $C E A$ carcinoembryonic antigen, $C R P C$-reactive protein, $O R$ odds ratio, $C l$ confidence interval

*P value $<0.05$ 
Table 4 ERAS nursing program and hospitalization satisfaction-related variables in LRC patients

\begin{tabular}{lllc}
\hline $\begin{array}{l}\text { Hospitalization } \\
\text { satisfaction-related } \\
\text { variables }\end{array}$ & \multicolumn{2}{l}{ ERAS nursing program } & P-value \\
\cline { 2 - 3 } & Yes & No & \\
\hline Number, n (\%) & $188(65.3)$ & $100(34.7)$ & - \\
Safety & $14.1 \pm 2.3$ & $13.9 \pm 2.5$ & 0.496 \\
Environment & $8.9 \pm 0.7$ & $8.8 \pm 1.0$ & 0.323 \\
Accessibility & $19.5 \pm 0.7$ & $19.4 \pm 0.8$ & 0.273 \\
Respect & $14.8 \pm 1.6$ & $14.5 \pm 1.9$ & 0.157 \\
Nursing technique & $4.9 \pm 0.6$ & $4.8 \pm 0.7$ & 0.205 \\
Comfortableness & $24.6 \pm 0.9$ & $24.2 \pm 1.2$ & $0.002^{*}$ \\
Health education & $9.6 \pm 0.6$ & $9.5 \pm 0.8$ & 0.313 \\
Communication & $14.4 \pm 1.1$ & $13.4 \pm 1.3$ & $<0.001^{*}$ \\
Emotional support & $4.8 \pm 0.4$ & $4.3 \pm 0.6$ & $<0.001^{*}$ \\
Participation in nursing & $14.7 \pm 0.9$ & $14.3 \pm 1.1$ & $0.001^{*}$ \\
Discharge and referral & $14.3 \pm 0.8$ & $14.2 \pm 1.0$ & 0.356 \\
\hline
\end{tabular}

ERAS enhanced recovery after surgery, $L R C$ low rectal cancer

${ }^{*} P$ value $<0.05$

Table 5 ERAS nursing program and prognosis-related variables in LRC patients

\begin{tabular}{lllc}
\hline \multirow{2}{*}{$\begin{array}{l}\text { Prognosis-related } \\
\text { variables }\end{array}$} & \multicolumn{2}{l}{ ERAS nursing program } & \multirow{2}{*}{$\boldsymbol{P}$-value } \\
\cline { 2 - 3 } & Yes & No & \\
\hline Number, n (\%) & $188(65.3)$ & $100(34.7)$ & - \\
Time to first exhaust (day) & $2.1 \pm 0.8$ & $2.3 \pm 0.7$ & $0.036^{*}$ \\
Time to defecation (day) & $3.3 \pm 0.9$ & $3.7 \pm 1.2$ & $0.002^{*}$ \\
LOS (day) & $6.9 \pm 1.3$ & $7.4 \pm 1.8$ & $0.007^{*}$ \\
SAS scores & $53.1 \pm 6.7$ & $51.4 \pm 5.0$ & $0.027^{*}$ \\
SDS scores & $52.3 \pm 5.5$ & $50.7 \pm 4.4$ & $0.005^{*}$ \\
GIQLI scores & - & - & - \\
Subjective symptoms & $65.4 \pm 5.7$ & $63.5 \pm 6.1$ & 0.009 \\
Physiological function & $19.1 \pm 2.3$ & $18.3 \pm 1.7$ & 0.002 \\
$\quad$ Mental health & $13.5 \pm 1.9$ & $12.9 \pm 1.6$ & 0.008 \\
Social function & $13.1 \pm 2.1$ & $12.5 \pm 1.8$ & 0.016 \\
\hline
\end{tabular}

ERAS enhanced recovery after surgery, $L R C$ low rectal cancer, $L O S$ length of stay, SAS self-rating anxiety scale, SDS self-rating depression scale, GIQLI Gastrointestinal Quality of Life Index

${ }^{*} P$ value $<0.05$

patients following ostomy surgery over two years [20]. Moreover, the incidences between the elective and emergency operation groups were quite similar [20]. Another study by Arumugam et al. has elaborated an incidence of $50.5 \%$ for one or more SRCs in a prospective study of 97 patients [21]. We considered that the differences in stoma location (colostomy or ileostomy), patient characteristics, surgical types, stoma nursing, and SRCs definitions were probably the main explanations for different incidences in different reports.
Various variables have been identified as independent risk factors for SRCs, including ASA grade [22], age, ileostomy, and loop stomas [23], male sex, and ileostomy creation [24]. Our results firstly demonstrated that ERAS nursing program was associated with decreased SRCs rates. A previous study by Forsmo et al. revealed that SRCs were not significantly different between ERAS and non-ERAS groups although with a tendency [25], which was not quite in accordance with our results. The stoma nursing has great impacts on the occurrence of SRCs, especially stoma infection and fecal dermatitis.

Based on our results, it appears that ERAS nursing program provided significant benefit to LRC patients undergoing abdominoperineal resection with sigmoidostomy. Our results demonstrated that the ERAS nursing program can be successfully implemented in a single-center municipal hospital. With the adoption of ERAS nursing program, patients were more likely to have better hospitalization satisfaction for the items of comfortableness, communication, emotional support, and participation in nursing. Furthermore, patients who underwent ERAS nursing program had an improved prognosis, which was manifested by earlier gastrointestinal function recovery (shorter time to first exhaust and defecation), shorter LOS, higher SDS and SAS scores, higher QOL (higher GIQLI scores). The decreased narcotic use in the ERAS program partially explains enhanced bowel functional recovery due to the well-known effects of narcotics [26]. Preoperative nutritional evaluation and support by ERAS nursing program can reduce the incidence of insulin resistance and postoperative hyperglycemia, and reduce preoperative anxiety. The improvement of nutritional status is widely reported to be associated with improved systemic immune function and better prognosis [27, 28]. Stoma formation is also a great threat to QOL for LRC patients due to the low acceptance rate, troublesome stoma nursing, and psychological burden. To some extent, the decreased SRCs rate correlates with increased QOL. The importance of guarantee a further rectal continence and function in patients who underwent ostomy is mandatory. But several others factors that could alter physiologic defecation are implied in fecal continence. Bocchini et al. [29] suggest the criteria for patients selection, evaluation of clinical presentation of defecatory disorders, appropriate education about concepts of pelvic anatomy and defecation physiology, and protocols of pelvic floor rehabilitation for defecation disorders, including fecal incontinence. Based on their reports, Brusciano et al. [30] propose a modified physiatric assessment (combination of chest, abdomen, vertebral column, and perineum) instead of solely on the function and integrity of the pelvic floor. Interestingly, Gambardella et al. [31] hold the same view that the muscular synergies evaluation (a 
clinical-physiatric evaluation) can serve as a predictive parameter to identify incontinent patients amenable for rehabilitation treatment. In summary, a systemic evaluation is recommended before pelvic floor rehabilitation for defecation disorders.

There are some limitations to this study. First, this is a single-center study with a relatively small sample size. Second, the retrospective nature and long-time inclusion period generate some uncontrollable biases. Third, the involved mechanisms remain unknown. Last, only LRC patients were enrolled and whether our conclusions apply to other stoma operations remain uncertain. Considering ERAS nursing program can reduce postoperative SRCs, improve the hospitalization satisfaction and QOL, it would be generally desirable.

\section{Conclusion}

In conclusion, ERAS nursing program was a protective factor for SRCs and associated with improved prognosis in LRC patients undergoing elective abdominoperineal resection with sigmoidostomy.

\begin{abstract}
Abbreviations
ERAS: Enhanced recovery after surgery; SRCs: Stoma-related complications; LRC: Low rectal cancer; QOL: Life of quality; CRC: Colorectal cancer; LOS: Length of stay; BMI: Body mass index; CEA: Carcinoembryonic antigen; NRS: Nutritional risk score; Hb: Hemoglobin; Alb: Albumin; WBC: White blood cell; Hct: Hematocrit; CRP: C-reactive protein; SAS: Self-rating anxiety scale; SDS: Self-rating depression scale; GIQLI: Gastrointestinal Quality of Life Index; SD: Standard deviation; IBD: Inflammatory bowel disease; OR: Odds ratio; Cl: Confidence interval.
\end{abstract}

\section{Acknowledgements}

Not applicable.

\section{Authors' contributions}

WLS, HGW, QC, and WZ: conception and design, data collection, statistical analysis and wrote the manuscript. YLG, GQF: study design, data collection, and study design. All authors read and approved the final manuscript.

\section{Funding}

The study was supported by the Taizhou Science and Technology Support Plan (social development) (No. TS201801), and Jiangsu Provincial Medical Youth Talent (QNRC 2016514).

\section{Availability of data and materials}

Please contact the corresponding author (Guogin Feng, email: fengguoqin. tz@outlook.com) for data requests.

\section{Ethics approval and consent to participate}

This study protocol was approved by the Medical Institutional Ethics Committee of Taizhou People's Hospital. All patients included were required to offer written informed consent.

\section{Consent for publication}

Not applicable.

\section{Competing interests}

The authors declare that they have no competing interests.

\section{Author details}

${ }^{1}$ Department of General Surgery, Taizhou People's Hospital, Taizhou, China.

${ }^{2}$ Department of Nursing, Taizhou People's Hospital, No. 366 Taihu Road,

Taizhou 225300, Jiangsu Province, China.

Received: 8 September 2020 Accepted: 22 November 2020

Published online: 04 December 2020

\section{References}

1. Bray F, Ferlay J, Soerjomataram I, Siegel RL, Torre LA, Jemal A. Global cancer statistics 2018: GLOBOCAN estimates of incidence and mortality worldwide for 36 cancers in 185 countries. CA Cancer J Clin. 2018;68(6):394-424.

2. Gu J, Chen N. Current status of rectal cancer treatment in China. Colorectal Dis. 2013;15(11):1345-50.

3. Gopall J, Shen XF, Cheng Y. Current status of laparoscopic total mesorectal excision. Am J Surg. 2012;203(2):230-41.

4. Simillis C, Baird DL, Kontovounisios C, Pawa N, Brown G, Rasheed S, Tekkis PP. A systematic review to assess resection margin status after abdominoperineal excision and pelvic exenteration for rectal cancer. Ann Surg. 2017;265(2):291-9.

5. Bafford AC, Irani JL. Management and complications of stomas. Surg Clin North Am. 2013;93(1):145-66.

6. Shabbir J, Britton DC. Stoma complications: a literature overview. Colorectal Dis. 2010;12(10):958-64.

7. Peeters KC, Tollenaar RA, Marijnen CA, Klein Kranenbarg E, Steup WH, Wiggers T, Rutten HJ, van de Velde CJ. Dutch Colorectal Cancer G: risk factors for anastomotic failure after total mesorectal excision of rectal cancer. $\mathrm{Br} J$ Surg. 2005;92(2):211-6.

8. Miyo M, Takemasa I, Ikeda M, Tujie M, Hasegawa J, Ohue M, Kato T, Mizushima T, Doki Y, Mori M. The influence of specific technical maneuvers utilized in the creation of diverting loop-ileostomies on stoma-related morbidity. Surg Today. 2017;47(8):940-50.

9. Phatak UR, Kao LS, You YN, Rodriguez-Bigas MA, Skibber JM, Feig BW, Nguyen S, Cantor SB, Chang GJ. Impact of ileostomy-related complications on the multidisciplinary treatment of rectal cancer. Ann Surg Oncol. 2014;21(2):507-12.

10. Kehlet H. Multimodal approach to control postoperative pathophysiology and rehabilitation. Br J Anaesth. 1997;78(5):606-17.

11. Khoo CK, Vickery CJ, Forsyth N, Vinall NS, Eyre-Brook IA. A prospective randomized controlled trial of multimodal perioperative management protocol in patients undergoing elective colorectal resection for cancer. Ann Surg. 2007;245(6):867-72.

12. Wind J, Polle SW, Fung Kon Jin PH, Dejong CH, von Meyenfeldt MF, Ubbink DT, Gouma DJ, Bemelman WA, Laparoscopy and/or Fast Track Multimodal Management Versus Standard Care Study G, Enhanced Recovery after Surgery G. Systematic review of enhanced recovery programmes in colonic surgery. Br J Surg. 2006;93(7):800-9.

13. Lau CS, Chamberlain RS. Enhanced recovery after surgery programs improve patient outcomes and recovery: a meta-analysis. World J Surg. 2017:41(4):899-913.

14. Charlson ME, Pompei P, Ales KL, MacKenzie CR. A new method of classifying prognostic comorbidity in longitudinal studies: development and validation. J Chronic Dis. 1987;40(5):373-83.

15. Kondrup J, Allison SP, Elia M, Vellas B, Plauth M. Educational, Clinical Practice Committee ESOP, Enteral N: ESPEN guidelines for nutrition screening 2002. Clin Nutr. 2003;22(4):415-21.

16. Zung WW. A rating instrument for anxiety disorders. Psychosomatics. 1971;12(6):371-9.

17. Eypasch E, Williams JI, Wood-Dauphinee S, Ure BM, Schmulling C, Neugebauer E, Troidl H. Gastrointestinal Quality of Life Index: development, validation and application of a new instrument. Br J Surg. 1995;82(2):216-22.

18. Bosio G, Pisani F, Fonti A, Scrocca A, Morandell C, Anselmi L, Antonini M, Militello G, Mastronicola G, Gasperini S, et al. Multicentre observational study on peristomal skin disorders. A proposal classification. G Chir. 2006;27(6-7):251-4. 
19. Makela JT, Turku PH, Laitinen ST. Analysis of late stomal complications following ostomy surgery. Ann Chir Gynaecol. 1997;86(4):305-10.

20. Robertson I, Leung E, Hughes D, Spiers M, Donnelly L, Mackenzie I, Macdonald A. Prospective analysis of stoma-related complications. Colorectal Dis. 2005;7(3):279-85.

21. Arumugam PJ, Bevan L, Macdonald L, Watkins AJ, Morgan AR, Beynon J, Carr ND. A prospective audit of stomas - analysis of risk factors and complications and their management. Colorectal Dis. 2003;5(1):49-52.

22. Nastro P, Knowles CH, McGrath A, Heyman B, Porrett TR, Lunniss PJ. Complications of intestinal stomas. Br J Surg. 2010;97(12):1885-9.

23. Park JJ, Del Pino A, Orsay CP, Nelson RL, Pearl RK, Cintron JR, Abcarian H. Stoma complications: the Cook County Hospital experience. Dis Colon Rectum. 1999;42(12):1575-80.

24. Arolfo S, Borgiotto C, Bosio G, Mistrangelo M, Allaix ME, Morino M. Preoperative stoma site marking: a simple practice to reduce stoma-related complications. Tech Coloproctol. 2018;22(9):683-7.

25. Forsmo HM, Pfeffer F, Rasdal A, Sintonen H, Korner H, Erichsen C. Preand postoperative stoma education and guidance within an enhanced recovery after surgery (ERAS) programme reduces length of hospital stay in colorectal surgery. Int J Surg. 2016;36(Pt A):121-6.

26. Kurz A, Sessler DI. Opioid-induced bowel dysfunction: pathophysiology and potential new therapies. Drugs. 2003;63(7):649-71.

27. Yilmaz A, Tekin SB, Bilici M, Yilmaz H. The Significance of Controlling Nutritional Status (CONUT) Score as a novel prognostic parameter in small cell lung cancer. Lung. 2020;198(4):695-704.
28. Matsubara T, Takamori S, Haratake N, Toyozawa R, Miura N, Shimokawa M, Yamaguchi M, Seto T, Takenoyama M. The impact of immune-inflammation-nutritional parameters on the prognosis of non-small cell lung cancer patients treated with atezolizumab. J Thorac Dis. 2020;12(4):1520-8.

29. Bocchini R, Chiarioni G, Corazziari E, Pucciani F, Torresan F, Alduini P, Bassotti G, Battaglia E, Ferrarini F, Galeazzi F, et al. Pelvic floor rehabilitation for defecation disorders. Tech Coloproctol. 2019;23(2):101-15.

30. Brusciano L, Gambardella C, Tolone S, Del Genio G, Terracciano G, Gualtieri G, Schiano di Visconte M, Docimo L. An imaginary cuboid: chest, abdomen, vertebral column and perineum, different parts of the same whole in the harmonic functioning of the pelvic floor. Tech Coloproctol. 2019;23(6):603-5.

31. Gambardella C, Brusciano L, Del Genio G, Tolone S, Terracciano G, Gualtieri G, Lucido FS, Docimo L. Predictive parameters to identify incontinent patients amenable for rehabilitation treatment: the muscular synergies evaluation. Arq Gastroenterol. 2019;56(4):452-3.

\section{Publisher's Note}

Springer Nature remains neutral with regard to jurisdictional claims in published maps and institutional affiliations.
Ready to submit your research? Choose BMC and benefit from:

- fast, convenient online submission

- thorough peer review by experienced researchers in your field

- rapid publication on acceptance

- support for research data, including large and complex data types

- gold Open Access which fosters wider collaboration and increased citations

- maximum visibility for your research: over $100 \mathrm{M}$ website views per year

At BMC, research is always in progress.

Learn more biomedcentral.com/submissions 